\title{
Accelerated ageing: from mechanism to therapy through animal models
}

\author{
Fernando G. Osorio · Álvaro J. Obaya • \\ Carlos López-Otín · José M. P. Freije
}

Received: 17 October 2008/ Accepted: 27 October 2008/Published online: 18 November 2008

(C) Springer Science+Business Media B.V. 2008

\begin{abstract}
Ageing research benefits from the study of accelerated ageing syndromes such as HutchinsonGilford progeria syndrome (HGPS), characterized by the early appearance of symptoms normally associated with advanced age. Most HGPS cases are caused by a mutation in the gene $L M N A$, which leads to the synthesis of a truncated precursor of lamin A known as progerin that lacks the target sequence for the metallopotease FACE-1/ZMPSTE24 and remains constitutively farnesylated. The use of Face-1/Zmpste24deficient mice allowed us to demonstrate that accumulation of farnesylated prelamin A causes severe abnormalities of the nuclear envelope, hyper-activation of p53 signalling, cellular senescence, stem cell dysfunction and the development of a progeroid phenotype. The reduction of prenylated prelamin A levels in genetically modified mice leads to a complete reversal of the progeroid phenotype,
\end{abstract}

F. G. Osorio · C. López-Otín · J. M. P. Freije ( $\)$

Departamento de Bioquímica y Biología Molecular, Facultad de Medicina, Instituto Universitario de Oncología, Universidad de Oviedo, 33006 Oviedo, Spain e-mail: jmpf@uniovi.es

\section{Á. J. Obaya}

Departamento de Biología Funcional (Fisiología), Facultad de Medicina, Instituto Universitario de Oncología, Universidad de Oviedo, 33006 Oviedo, Spain suggesting that inhibition of protein farnesylation could represent a therapeutic option for the treatment of progeria. However, we found that both prelamin A and its truncated form progerin can undergo either farnesylation or geranylgeranylation, revealing the need of targeting both activities for an efficient treatment of HGPS. Using Face-1/Zmpste24-deficient mice as model, we found that a combination of statins and aminobisphosphonates inhibits both types of modifications of prelamin A and progerin, improves the ageing-like symptoms of these mice and extends substantially their longevity, opening a new therapeutic possibility for human progeroid syndromes associated with nuclear-envelope defects. We discuss here the use of this and other animal models to investigate the molecular mechanisms underlying accelerated ageing and to test strategies for its treatment.

Keywords Proteases - Tumor suppression Cancer - Isoprenylation - Alternative splicing · Stem cell

\section{Introduction}

Ageing, the progressive and irreversible loss of physiological integrity, is an extremely complex process whose molecular basis remain incompletely understood (Kirkwood 2005). Several human 
illnesses, known as segmental progeroid syndromes, are characterized by the early development of multiple biological alterations normally associated with advanced age. Even though these rare and dramatic conditions only partially recapitulate normal ageing, their study has the potential of rendering valuable information on the molecular mechanisms implicated in the ageing process (Ramirez et al. 2007). Moreover, the development of animal models that phenocopy these syndromes can provide experimental systems useful to investigate the basis of particular pathologies associated with ageing (atherosclerosis, osteoporosis, osteoarthritis, cancer) and to perform preclinical testing of therapeutic strategies against these alterations.

Most human syndromes of accelerated ageing are caused by one of two major mechanisms: defects in DNA repair systems and alterations in the nuclear lamina. The best understood progeroid syndrome due to a DNA repair defect is Werner syndrome (WS), also known as progeria of the adult. WS patients show a wide array of symptoms of premature ageing, which emerge at puberty and include early growth stop, bilateral cataracts, grey hair, scleroderma-like skin changes, subcutaneous calcification, arteriosclerosis, diabetes mellitus, a prematurely aged facies and a high incidence of cancer. Most WS do not live past 50 years (Cox and Faragher 2007). Typical cases of WS are caused by null mutations in $W R N$, a gene coding for a protein of the RecQ family with helicase and exonuclease activities that plays important roles in homologydependent recombination repair and telomere maintenance (Yu et al. 1996). A Wrn-knockout mouse model recapitulates the alterations observed in WS patients at the molecular and cellular levels but, strikingly, Wrn deficiency does not causes an accelerated ageing phenotype in mice (Lombard et al. 2000). In contrast, progeroid symptoms closely recapitulating WS develop in double-mutant mice lacking both Wrn and telomerase activity, revealing the critical role of Wrn in telomere biology and its relevance for the progeroid phenotypes caused by WRN deficiency (Chang et al. 2004; Multani and Chang 2007). These findings indicate that mice and humans may show different sensitivity to progeroidcausing alterations, and these differences have to be carefully taken in consideration to interpret results derived from the use of murine models.
Hutchinson-Gilford progeria syndrome (HGPS), also known as progeria of childhood, is the best known accelerated ageing syndrome caused by defects of the nuclear envelope. HGPS is characterized by shortened lifespan, growth impairment, sclerotic skin, early hair loss, aged-facies, decreased joint mobility and cardiovascular problems (Hennekam 2006; Merideth et al. 2008; Pereira et al. 2008). Most HGPS cases are caused by a silent mutation in the LMNA gene, which encodes two components of the nuclear envelope, the lamins A and C. Lamin A is synthesized as a precursor known as prelamin $\mathrm{A}$, which undergoes a series of post-translational modifications including farnesylation, proteolytic removal of the C-terminal tripeptide, carboxyl methylation of the prenylated cysteine residue and finally, the excision of the 15-residue farnesylated peptide. The mutation present in HGPS patients activates a cryptic splicing site, and leads to the synthesis of a prelamin A isoform known as progerin or LA $\Delta 50$, which lacks a 50-residue long fragment containing the target sequence for the final proteolytic step and consequently remains constitutively farnesylated (De Sandre-Giovannoli et al. 2003; Eriksson et al. 2003).

The use of genetically modified mice allowed us to identify the zinc metalloprotease FACE-1 (also known as ZMPSTE24) as the enzyme responsible for the final proteolytic step during lamin A posttranslational maturation (Pendas et al. 2002). Accordingly, Face-1/Zmpste24-deficient mice accumulate farnesylated prelamin A at the nuclear envelope and phenocopy human HGPS, providing a valuable animal model for the study of this pathology (Pendas et al. 2002; Bergo et al. 2002; Cadinanos et al. 2005; de Carlos et al. 2008). Using transcriptional profiling on tissues from this knockout model, we found that hyperactivation of p53 signalling plays a key role in the accelerated ageing phenotype, which is partially reversed by p53 deficiency (Varela et al. 2005). Moreover, the use of genetic approaches revealed that lowering the prelamin A levels results in a total rescue of this mouse model from the accelerated ageing condition (Varela et al. 2005; Fong et al. 2004; 2006b). Based on this information, we have performed preclinical studies to test anti-progeria pharmacological approaches aimed to prevent the accumulation of prenylated prelamin A isoforms (Varela et al. 2008). 


\section{Zmpste24-deficient mice as a model of accelerated ageing}

The zinc metalloprotease FACE-1/ZMPSTE24 is an integral membrane protein highly conserved throughout evolution that shows widespread expression in mammalian tissues (Freije et al. 1999; Tam et al. 1998; Kumagai et al. 1999; Cadinanos et al. 2003). To investigate the biological roles of this enzyme, we used gene targeting to generate Zmpste $24^{-/-}$mice (Pendas et al. 2002). Zmpste24deficient mice are apparently normal at birth, but they show a striking accumulation of prelamin A at the nuclear envelope, which leads to frequent nuclear abnormalities at the cellular level. In turn, these molecular and cellular alterations lead to the development of severe age-related abnormalities at the organismal level, including loss of subcutaneous fat, reduced mobility due to skeletal and muscular defects, hair loss and metabolic alterations (Pendas et al. 2002; Bergo et al. 2002).

The subsequent identification of progerin as the molecular cause of HGPS (De Sandre-Giovannoli et al. 2003; Eriksson et al. 2003) revealed that a proteolytic defect explains the resemblance of the phenotype shown by Zmpste24-deficient mice to the clinical features characteristic of HGPS. Thus, whereas in Zmpste $24^{-1-}$ mice prenylated prelamin A accumulates due to the absence of the protease responsible for removing the $\mathrm{C}$-terminal peptide that contains the prenyl-cysteine residue, the accumulation of progerin in HGPS is a consequence of the loss of the Zmpste24 target site in this prelamin A truncated isoform (Fig. 1). In addition, mutations in ZMPSTE24 have also been found in several human progeroid syndromes, such as mandibuloacral dysplasia (MAD) and restrictive dermopathy (RD) (Agarwal et al. 2003; Navarro et al. 2005; Shackleton et al. 2005; Sander et al. 2008), providing additional support to the relevance of Zmpste $24^{-/-}$mice as a model for studying accelerated ageing. Interestingly, accumulation of prenylated progerin has also been associated with normal ageing, a finding that expands considerably the interest of Zmpste24-deficient mice and other related animal models of progeria (Scaffidi and Misteli 2006).

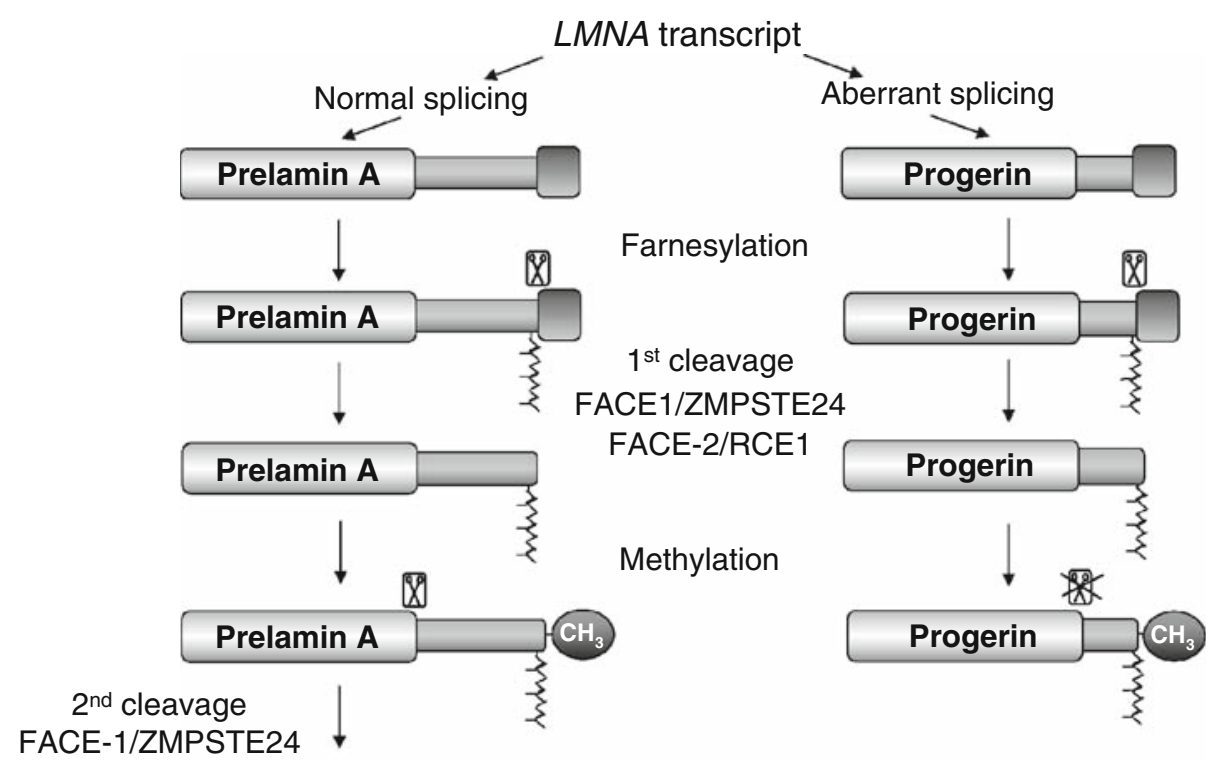

Lamin A

Fig. 1 Lamin A and progerin processing. In Face-1/Zmpste24-deficient mice, the absence of this metalloprotease causes the accumulation of farnesylated prelamin A. In HGPS cells, a $\mathrm{C}-\mathrm{T}$ transition in the LMNA gene introduces a cryptic splicing site, leading to the synthesis of an alternatively spliced mRNA that encodes progerin, a truncated prelamin A variant that lacks the Face-1/Zmpste24 target sequence and remains constitutively farnesylated 


\section{Transcriptional and biochemical alterations in $\mathrm{Zmpste}^{24^{-/-}}$progeroid mice}

The accumulation of immature prenylated lamin forms (prelamin A and progerin) at the nuclear envelope produces severe alterations in its architecture, leading to frequent nuclear blebbing and fragmentation (Pendas et al. 2002). These alterations in nuclear architecture induced by prelamin A and progerin have also been linked to a defective recruitment of DNA repair factors and increased genomic instability (Liu et al. 2005). In agreement with these findings, transcriptional profiling of tissues from Zmpste24deficient mice revealed a clear up-regulation of p53 target genes, which becomes more pronounced as the progeroid phenotype of these animals becomes more dramatic (Varela et al. 2005). Upregulation of p53 pathway seems to underlie the reduced proliferative capacity of adult Zmpste24-deficient fibroblasts and play a causal role in the accelerated ageing phenotype associated to prelamin A accumulation, as p53 deficiency results in a delay of the accelerated ageing process shown by Zmpste $24^{-1-}$ mice (Varela et al. 2005). The results derived from the use of this animal model are, at least partially, translatable to human as p53 activation has been detected in cells from RD and HGPS patients (Liu et al. 2006). Moreover, it has been reported that targeting progerin accumulation reverts overexpression of p53 targets in aged individuals (Scaffidi and Misteli 2006).

The persistence of ageing-related alterations in Zmpste24-deficient mice lacking p53 reveals that additional molecular mechanisms are involved in the phenotype developed by these mice. Some of these additional alterations seem to be part of a metabolic pro-survival strategy turned into a pro-ageing mechanism due to its chronic activation. This is the case of autophagy, the major lysosomal pathway for the turnover of cytoplasmic components. In contrast to the known decline of autophagy that results in the accumulation of damaged macromolecules during physiological ageing, progeroid mouse models exhibit a pronounced activation of autophagic proteolysis (Marino et al. 2008; Marino and Lopez-Otin 2008). Remarkably, we found that this alteration is also shared by other progeroid models which show alterations in DNA repair systems. Increased autophagy in Zmpste24-deficient mice is caused by a reduced activity of mTOR, which, in turn, is linked to activation of LKB1-AMPK signalling, transcriptional changes in key genes for lipid and glucose metabolism regulation, hypoglycemia and other alterations in serum factors such as leptin, insulin and adiponectin (Marino et al. 2008). These signalling alterations and their associated metabolic changes have been related to situations prolonging lifespan, such as calorie restriction (Vijg and Campisi 2008), and could be part of a response triggered by the nuclear abnormalities present in Zmpste24-deficient animals. However, the chronic activation of this pro-survival strategy can result in a pro-ageing effect that contributes to the progeroid phenotype of these mice (Marino et al. 2008). The apparently paradoxical activation of prolongevity mechanisms in Zmpste24-deficient progeroid mice confers this animal model a special interest as a tool for investigating the potential undesirable effects of nutritional and pharmacological anti-ageing interventions and to develop complementary treatments to mitigate them.

\section{Altered stem-cell biology in Zmpste24-deficient mice}

The molecular alterations discussed in the previous paragraphs could have a special relevance on the accelerated ageing of Zmpste24-deficient organs and tissues if these alterations affect the stem cells responsible for their regenerative capacity. Physiological ageing has been associated with changes in the number and functionality of somatic stem cells (Campisi 2005; Rando 2006). Thus, the availability of Zmpste24-deficient mice allowed us to test the hypothesis that stem cell dysfunction could be similarly involved in accelerated ageing conditions. Using in vivo labelling, we observed a significant increase in the number of Zmpste24-deficient epidermal stem cells, accompanied by a reduction in their proliferative potential and an increase in apoptosis in their supporting cells (Espada et al. 2008). These abnormalities are associated with defects in signalling pathways such as Wnt- $\beta$-catenin, implicated in the functional regulation of epidermal stem cells, and microphtalmia transcription factor (Mitf), a master regulator of melanocyte stem cells (Espada et al. 2008). Remarkably, an independent but highly complementary study demonstrated in vitro that progerin interferes with the biology of human mesenchymal 
stem cells (hMSCs) (Scaffidi and Misteli 2008), revealing that the stem cell dysfunction identified in the Zmpste24-deficient animal model also plays a role in human progeria. Taken together, these results suggest that the progeroid model of Zmpste24 protease deficiency could be used for in vivo testing of cell-based anti-progeroid therapies.

\section{Zmpste24-deficient mice as a model for developing therapies against accelerated ageing}

To investigate the role of unprocessed prelamin A in accelerated ageing, we designed a breeding program aimed at developing Zmpste24-deficient mice with reduced levels of prelamin A. This approach revealed that Zmpste $24^{-1-}$ Lmna $^{+/-}$mice show significantly reduced prelamin A levels and do not develop progeroid symptoms, demonstrating the role of prelamin A accumulation as the primary cause of the progeroid phenotype associated to Zmpste24 deficiency (Varela et al. 2005; Fong et al. 2004). Parallel studies carried out using HGPS fibroblasts showed that accumulation of progerin plays an equivalent causal role in the cellular phenotype developed by these cells (Scaffidi and Misteli 2005). Moreover, these studies pointed to the reduction of progerin levels as an appropriate target to develop therapeutic approaches against HGPS. Surprisingly, genetic ablation of farnesyltransferase after birth does not lead to an accelerated ageing phenotype in mice, even though prenylation of prelamin $\mathrm{A}$ is a required step for its subsequent proteolytic processing (Mijimolle et al. 2005). This observation suggested that the toxicity of lamin A precursors depends on their prenylation status and pointed to the use of farnesyltransferase inhibitors (FTIs) as a potential treatment for these conditions (Cadinanos et al. 2005). Thus, several studies revealed that FTI treatment was capable of improving the nuclear abnormalities in several cellular models of progeria (Glynn and Glover 2005; Mallampalli et al. 2005; Yang et al. 2005; Toth et al. 2005; Capell et al. 2005).

Some in vivo benefit was also described in Zmpste24-deficient mice (Fong et al. 2006a), although further studies revealed that this treatment only produced a marginal $(5 \%)$ reduction in prelamin A processing (Young et al. 2006). This low effectiveness at the molecular level could be explained by an alternative prenylation of prelamin A by geranylgeranyltransferase type I under FTI treatment (Fig. 2), as reported for other prenylated proteins such as K-Ras (Whyte et al. 1997). To address this question, we tested the effect of FTIs, alone or in combination with geranylgeranyltransferase inhibitors (GGTIs), on prelamin A processing in human cells. This approach revealed a synergistic action of FTIs and GGTIs, suggesting that prelamin A can be geranylgeranylated in the setting of farnesyltransferase inhibition (Varela et al. 2008). Furthermore, the use of mass
Fig. 2 Therapeutic options to prevent the accumulation of prenylated progerin. The possibilities discussed include compounds acting on the mevalonate pathway such as statins, and aminobisphosphonates (N-BPs), farnesyltransferase inhibitors (FTI), geranylgeranyltransferase inhibitors (GGTI), and agents designed to block the alternative splicing event that leads to progerin synthesis such as small drugs or specific antisense oligonucleotides (AS oligos)

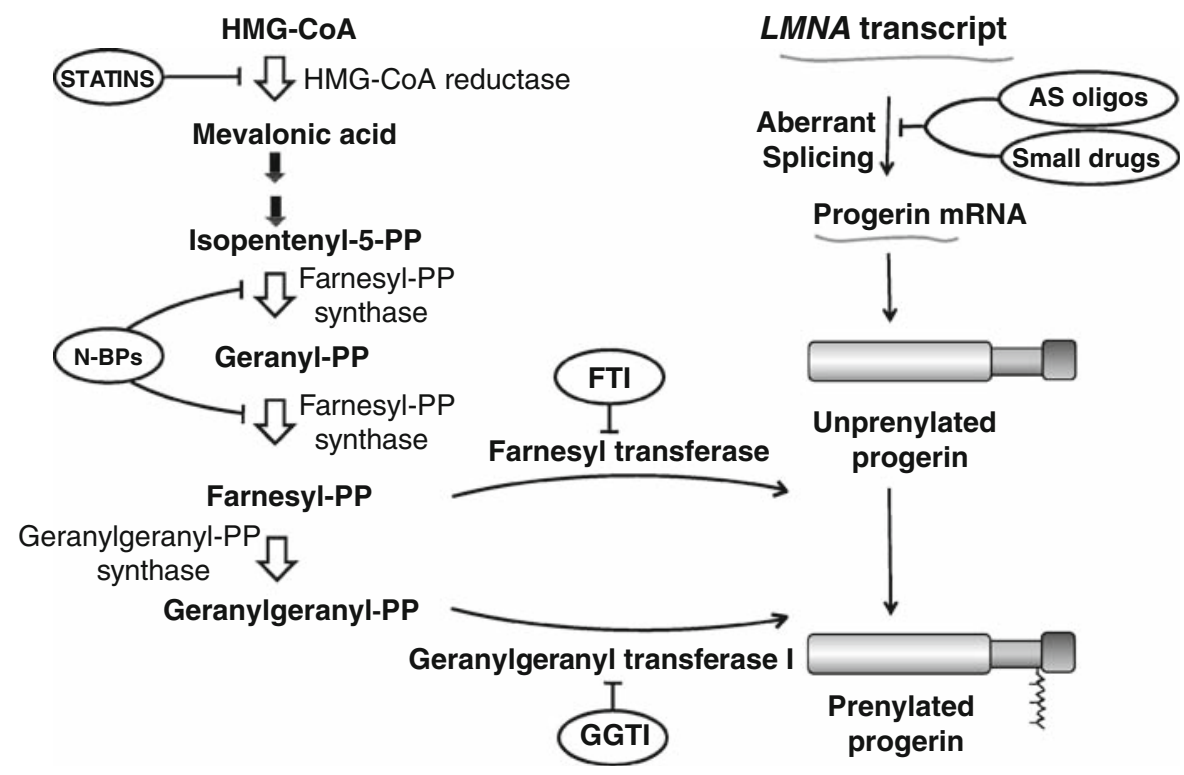


spectrometry analysis of prelamin A derived from FTI-treated Zmpste24-deficient fibroblasts and progerin derived from HGPS cells provided direct evidence that these proteins are alternatively geranylgeranylated when farnesylation is inhibited, which could explain the low efficiency of FTIs in ameliorating the phenotypes of progeroid mouse models. In agreement with these findings, a combination of statins and aminobisphosphonates blocking several steps of the farnesyl pyrophosphate and geranylgeranyl pyrophosphate biosynthetic pathway, efficiently inhibited both farnesylation and geranylgeranylation of progerin and prelamin A and improved the ageing-like phenotypes of Zmpste24-deficient animals (Varela et al. 2008). Based on these results, a clinical trial to test this combination of drugs for the treatment of progeria has been recently approved.

\section{Additional animal models for studying progeroid laminopathies}

As discussed above, Zmpste24-deficient mice have constituted a crucial tool to explore the mechanisms underlying progeroid laminopathies and to design therapeutic approaches for their treatment, whereas other related animal models have confirmed or extended these findings. Thus, the so-called "lamin C only" mice, carrying a Lmna allele which fails to undergo the lamin A-specific splicing, have revealed that lamin A is dispensable in mice (Fong et al. 2006b). These studies have also provided additional evidence of the causative role of prelamin A accumulation in the progeroid phenotype of Zmpste24-deficient animals, thereby supporting the conclusions raised by using Lmna $^{+-}$animals (Varela et al. 2005). Similarly, knock-in mice carrying a "progerin-only" Lmna allele phenocopy many of the abnormalities present in Zmpste24-deficient animals, and show a modest amelioration upon FTI treatment (Yang et al. 2005; 2006; 2008b). Even though a knock-in mouse expressing the same mutant protein present in HGPS patients would be expected to be the ideal model for this disease, the reported "progerin-only" mice present some problems. First, the maintenance of the model has to rely on chimeras due to the fertility problems of the mice heterozygous for this mutation, making very difficult to generate enough animals to reach statistical significance in any study. Second, the expression levels of progerin and the ratio progerin/normal lamins $\mathrm{A} / \mathrm{C}$ are different to those observed in HGPS patients. Third, the genomic sequences involved in the alternative splicing responsible for HGPS are absent in the "progerinonly" allele, precluding the use of this model to test therapeutic approaches such as specific oligonucleotides or small drugs designed to target this pathogenic splicing event (Fig. 2) (Scaffidi and Misteli 2005).

Remarkably, the recent information derived from two new Lmna knock-in models has validated important aspects of previous work with Zmpste24deficient mice. Thus, Yang et al. have reported that mice with a Lmna allele encoding a non-prenylatable progerin (nHG) show substantially milder abnormalities and an extended longevity as compared to mice with a prenylatable "progerin-only" allele (Yang et al. 2008a). The amelioration observed in these mice is comparable to that obtained in Zmpste24deficient animals treated with a combination of statins and aminobisphosphonates capable of blocking efficiently prelamin A prenylation (Varela et al. 2008). Moreover, this study has revealed that accumulation of non-prenylated progerin also causes accelerated ageing symptoms, suggesting that therapeutic approaches based on blocking progerin prenylation may require complementary interventions to reach a complete efficacy (Yang et al. 2008a). Finally, Davies et al., have described a Lmna knockin allele encoding a geranylgeranylated progerin that also causes a progeroid phenotype, providing a formal demonstration of the toxicity of this alternatively modified protein (Davies et al. 2008), and supporting the conclusions derived from mass spectrometry analysis of FTI-treated Zmpste24-deficient and HGPS cells (Varela et al. 2008).

Besides the knock-in mice discussed above, several transgenic models of progeria have also been developed. Thus, Varga et al. (2006) have developed a transgenic mouse strain carrying a $164-\mathrm{kb}$ bacterial artificial chromosome containing an LMNA allele with the HGPS point mutation. These transgenic mice suffer important vascular alterations, which constitute the most common cause of death for HGPS patients, but they do not show any other progeroid phenotype, limiting their utility as a model of accelerated ageing. The epidermal alterations characteristic of progeroid laminopathies have prompted other authors to develop transgenic models expressing progerin in keratinocytes. Thus, mice expressing FLAG-tagged human 
progerin under the control of a keratin 14 promoter have abnormal morphology of keratinocyte nuclei, but they do not show any skin pathology (Wang et al. 2008). In contrast, doxycycline-regulated transgenic mice (tet-off) expressing human progerin under a keratin 5 promoter develop dental and skin alterations which are reversed upon suppression of the transgene expression (Sagelius et al. 2008a; b). The reasons for the apparent discrepancies between these two works are currently unclear, and can be related to subtle differences in the spatio-temporal pattern of expression of the transgene in both systems.

\section{Future models and perspectives}

As discussed above, several knock-out, knock-in and transgenic animal models have been used to investigate the role of alterations in the prelamin A posttranslational maturation in accelerated ageing and to develop therapeutic strategies against progeroid symptoms. All these models have provided valuable information on the biological relevance of prelamin A maturation. However, important questions remain to be answered, concerning aspects such as the relative contribution to the progeroid phenotype of cell-autonomous versus systemic alterations. The investigation of this important aspect will be facilitated by the use of inducible systems, similar to the tet-off system used to direct progerin expression to keratinocytes, but designed to express prelamin A or progerin in other organs and tissues with a decisive role in the clinical manifestations of accelerated and physiological ageing. New animal models are also required to test in vivo anti-progeria therapies, since none of the currently available mice are appropriate to test approaches such as those targeting the alternative splicing responsible for progerin production. Lmna knock-in mice carrying an allele with the complete genomic sequence involved in this splicing event will probably be the ideal model to investigate this type of interventions. Finally, animal models will predictably play a key role as tools for investigating not only the implications of the Zmpste24/lamin A system in accelerated and physiological ageing, but also in other important processes such as cancer. In this regard, recent work illustrates the relevance of this system in cancer and supports its interest as target of anti-cancer therapies (Willis et al. 2008), an aspect that will be the subject of future studies based on the use of currently available and newly developed animal models. In summary, the use of genetically modified mice has allowed investigating the molecular mechanisms underlying progeroid laminopathies and has lead, in a very short period of time, to clinical trials for testing rationally designed treatments against these dramatic diseases. Nevertheless, important aspects remain unsolved that will require further investigations based on currently available and newly developed animal models.

Acknowledgments We thank I. Varela, X.S. Puente, J. Cadiñanos and G. Velasco for helpful comments and advice. This work was supported by grants from Ministerio de Educación y Ciencia-Spain, Fundación "La Caixa", Fundación "M. Botín", and the European Union (FP6 CancerDegradome and FP7 Microenvimet). The Instituto Universitario de Oncología is supported by Obra Social Cajastur-Asturias.

\section{References}

Agarwal AK, Fryns JP, Auchus RJ, Garg A (2003) Zinc metalloproteinase, ZMPSTE24, is mutated in mandibuloacral dysplasia. Hum Mol Genet 12:1995-2001. doi: $10.1093 / \mathrm{hmg} / \mathrm{ddg} 213$

Bergo MO, Gavino B, Ross J, Schmidt WK, Hong C, Kendall LV, Mohr A, Meta M, Genant H, Jiang Y, Wisner ER, Van Bruggen N, Carano RA, Michaelis S, Griffey SM, Young SG (2002) Zmpste24 deficiency in mice causes spontaneous bone fractures, muscle weakness, and a prelamin A processing defect. Proc Natl Acad Sci USA 99:13049-13054. doi:10.1073/pnas.192460799

Cadinanos J, Schmidt WK, Fueyo A, Varela I, Lopez-Otin C, Freije JM (2003) Identification, functional expression and enzymic analysis of two distinct $\mathrm{CaaX}$ proteases from Caenorhabditis elegans. Biochem J 370:1047-1054. doi: 10.1042/BJ20021514

Cadinanos J, Varela I, Lopez-Otin C, Freije JM (2005) From immature lamin to premature aging: molecular pathways and therapeutic opportunities. Cell Cycle 4:1732-1735

Campisi J (2005) Senescent cells, tumor suppression, and organismal aging: good citizens, bad neighbors. Cell 120:513-522. doi:10.1016/j.cell.2005.02.003

Capell BC, Erdos MR, Madigan JP, Fiordalisi JJ, Varga R, Conneely KN, Gordon LB, Der CJ, Cox AD, Collins FS (2005) Inhibiting farnesylation of progerin prevents the characteristic nuclear blebbing of Hutchinson-Gilford progeria syndrome. Proc Natl Acad Sci USA 102:1287912884. doi:10.1073/pnas.0506001102

Chang S, Multani AS, Cabrera NG, Naylor ML, Laud P, Lombard D, Pathak S, Guarente L, DePinho RA (2004) Essential role of limiting telomeres in the pathogenesis of Werner syndrome. Nat Genet 36:877-882. doi:10.1038/ng1389

Cox LS, Faragher RG (2007) From old organisms to new molecules: integrative biology and therapeutic targets in 
accelerated human ageing. Cell Mol Life Sci 64:26202641. doi:10.1007/s00018-007-7123-x

Davies BS, Yang SH, Farber E, Lee R, Buck SB, Andres DA, Peter Spielmann H, Agnew BJ, Tamanoi F, Fong LG, Young SG (2008) Increasing the length of progerin's isoprenyl anchor does not worsen bone disease or survival in mice with Hutchinson-Gilford progeria syndrome. J Lipid Res. doi:10.1194/jlr.M800424-JLR200

de Carlos F, Varela I, Germana A, Montalbano G, Freije J, Vega J, López-Otin C, Cobo J (2008) Microcephalia with mandibular and dental dysplasia in adult Zmpste24 deficient mice. J Anat 213:509-519. doi:10.1111/j.1469-7580. 2008.00970.x

De Sandre-Giovannoli A, Bernard R, Cau P, Navarro C, Amiel J, Boccaccio I, Lyonnet S, Stewart CL, Munnich A, Le Merrer M, Levy N (2003) Lamin A truncation in Hutchinson-Gilford progeria. Science 300:2055. doi:10.1126/ science. 1084125

Eriksson M, Brown WT, Gordon LB, Glynn MW, Singer J, Scott L, Erdos MR, Robbins CM, Moses TY, Berglund P, Dutra A, Pak E, Durkin S, Csoka AB, Boehnke M, Glover TW, Collins FS (2003) Recurrent de novo point mutations in lamin A cause Hutchinson-Gilford progeria syndrome. Nature 423:293-298. doi:10.1038/nature01629

Espada J, Varela I, Flores I, Ugalde AP, Cadinanos J, Pendas AM, Stewart CL, Tryggvason K, Blasco MA, Freije JM, Lopez-Otin C (2008) Nuclear envelope defects cause stem cell dysfunction in premature-aging mice. J Cell Biol 181:27-35. doi:10.1083/jcb.200801096

Fong LG, Ng JK, Meta M, Cote N, Yang SH, Stewart CL, Sullivan T, Burghardt A, Majumdar S, Reue K, Bergo MO, Young SG (2004) Heterozygosity for Lmna deficiency eliminates the progeria-like phenotypes in Zmpste24-deficient mice. Proc Natl Acad Sci USA 101:18111-18116. doi:10.1073/pnas.0408558102

Fong LG, Frost D, Meta M, Qiao X, Yang SH, Coffinier C, Young SG (2006a) A protein farnesyltransferase inhibitor ameliorates disease in a mouse model of progeria. Science 311:1621-1623. doi:10.1126/science.1124875

Fong LG, Ng JK, Lammerding J, Vickers TA, Meta M, Cote N, Gavino B, Qiao X, Chang SY, Young SR, Yang SH, Stewart CL, Lee RT, Bennett CF, Bergo MO, Young SG (2006b) Prelamin A and lamin A appear to be dispensable in the nuclear lamina. J Clin Invest 116:743-752. doi: 10.1172/JCI27125

Freije JM, Blay P, Pendas AM, Cadinanos J, Crespo P, LopezOtin C (1999) Identification and chromosomal location of two human genes encoding enzymes potentially involved in proteolytic maturation of farnesylated proteins. Genomics 58:270-280. doi:10.1006/geno.1999.5834

Glynn MW, Glover TW (2005) Incomplete processing of mutant lamin A in Hutchinson-Gilford progeria leads to nuclear abnormalities, which are reversed by farnesyltransferase inhibition. Hum Mol Genet 14:2959-2969. doi:10.1093/hmg/ddi326

Hennekam RC (2006) Hutchinson-Gilford progeria syndrome: review of the phenotype. Am J Med Genet A 140:26032624. doi:10.1002/ajmg.a.31346

Kirkwood TB (2005) Understanding the odd science of aging. Cell 120:437-447. doi:10.1016/j.cell.2005.01.027
Kumagai H, Kawamura Y, Yanagisawa K, Komano H (1999) Identification of a human cDNA encoding a novel protein structurally related to the yeast membrane-associated metalloprotease, Ste24p. Biochim Biophys Acta 1426: 468-474

Liu B, Wang J, Chan KM, Tjia WM, Deng W, Guan X, Huang JD, Li KM, Chau PY, Chen DJ, Pei D, Pendas AM, Cadinanos J, Lopez-Otin C, Tse HF, Hutchison C, Chen J, Cao Y, Cheah KS, Tryggvason K, Zhou Z (2005) Genomic instability in laminopathy-based premature aging. Nat Med 11:780-785. doi:10.1038/nm1266

Liu Y, Rusinol A, Sinensky M, Wang Y, Zou Y (2006) DNA damage responses in progeroid syndromes arise from defective maturation of prelamin A. J Cell Sci 119:46444649. doi: $10.1242 /$ jcs. 03263

Lombard DB, Beard C, Johnson B, Marciniak RA, Dausman J, Bronson R, Buhlmann JE, Lipman R, Curry R, Sharpe A, Jaenisch R, Guarente L (2000) Mutations in the WRN gene in mice accelerate mortality in a p53-null background. Mol Cell Biol 20:3286-3291. doi:10.1128/ MCB.20.9.3286-3291.2000

Mallampalli MP, Huyer G, Bendale P, Gelb MH, Michaelis S (2005) Inhibiting farnesylation reverses the nuclear morphology defect in a HeLa cell model for HutchinsonGilford progeria syndrome. Proc Natl Acad Sci USA 102:14416-14421. doi:10.1073/pnas.0503712102

Marino G, Lopez-Otin C (2008) Autophagy and aging: new lessons from progeroid mice. Autophagy 4:807-809

Marino G, Ugalde AP, Salvador-Montoliu N, Varela I, Quiros PM, Cadinanos J, van der Pluijm I, Freije JM, Lopez-Otin C (2008) Premature aging in mice activates a systemic metabolic response involving autophagy induction. Hum Mol Genet 17:2196-2211. doi:10.1093/hmg/ddn120

Merideth MA, Gordon LB, Clauss S, Sachdev V, Smith AC, Perry MB, Brewer CC, Zalewski C, Kim HJ, Solomon B, Brooks BP, Gerber LH, Turner ML, Domingo DL, Hart TC, Graf J, Reynolds JC, Gropman A, Yanovski JA, Gerhard-Herman M, Collins FS, Nabel EG, Cannon RO 3rd, Gahl WA, Introne WJ (2008) Phenotype and course of Hutchinson-Gilford progeria syndrome. N Engl J Med 358:592-604. doi:10.1056/NEJMoa0706898

Mijimolle N, Velasco J, Dubus P, Guerra C, Weinbaum CA, Casey PJ, Campuzano V, Barbacid M (2005) Protein farnesyltransferase in embryogenesis, adult homeostasis, and tumor development. Cancer Cell 7:313-324. doi: 10.1016/j.ccr.2005.03.004

Multani AS, Chang S (2007) WRN at telomeres: implications for aging and cancer. J Cell Sci 120:713-721. doi: $10.1242 /$ jcs.03397

Navarro CL, Cadinanos J, De Sandre-Giovannoli A, Bernard R, Courrier S, Boccaccio I, Boyer A, Kleijer WJ, Wagner A, Giuliano F, Beemer FA, Freije JM, Cau P, Hennekam RC, Lopez-Otin C, Badens C, Levy N (2005) Loss of ZMPSTE24 (FACE-1) causes autosomal recessive restrictive dermopathy and accumulation of lamin A precursors. Hum Mol Genet 14:1503-1513. doi:10.1093/hmg/ddi159

Pendas AM, Zhou Z, Cadinanos J, Freije JM, Wang J, Hultenby K, Astudillo A, Wernerson A, Rodriguez F, Tryggvason K, Lopez-Otin C (2002) Defective prelamin A processing and muscular and adipocyte alterations in 
Zmpste24 metalloproteinase-deficient mice. Nat Genet 31:94-99

Pereira S, Bourgeois P, Navarro C, Esteves-Vieira V, Cau P, De Sandre-Giovannoli A, Levy N (2008) HGPS and related premature aging disorders: from genomic identification to the first therapeutic approaches. Mech Ageing Dev 129:449-459. doi:10.1016/j.mad.2008.04.003

Ramirez CL, Cadinanos J, Varela I, Freije JM, Lopez-Otin C (2007) Human progeroid syndromes, aging and cancer: new genetic and epigenetic insights into old questions. Cell Mol Life Sci 64:155-170. doi:10.1007/s00018-006-6349-3

Rando TA (2006) Stem cells, ageing and the quest for immortality. Nature 441:1080-1086. doi:10.1038/nature04958

Sagelius H, Rosengardten Y, Hanif M, Erdos MR, Rozell B, Collins FS, Eriksson M (2008a) Targeted transgenic expression of the mutation causing Hutchinson-Gilford progeria syndrome leads to proliferative and degenerative epidermal disease. J Cell Sci 121:969-978. doi:10.1242/ jes.022913

Sagelius H, Rosengardten Y, Schmidt E, Sonnabend C, Rozell B, Eriksson M (2008b) Reversible phenotype in a mouse model of Hutchinson-Gilford progeria syndrome. J Med Genet. doi:10.1136/jmg.2008.060772

Sander CS, Salman N, van Geel M, Broers JL, Al-Rahmani A, Chedid F, Hausser I, Oji V, Al Nuaimi K, Berger TG, Verstraeten VL (2008) A newly identified splice site mutation in ZMPSTE24 causes restrictive dermopathy in the middle East. $\mathrm{Br} J$ Dermatol 159:961-967. doi: 10.1111/j.1365-2133.2008.08772.x

Scaffidi P, Misteli T (2005) Reversal of the cellular phenotype in the premature aging disease Hutchinson-Gilford progeria syndrome. Nat Med 11:440-445. doi:10.1038/nm1204

Scaffidi P, Misteli T (2006) Lamin A-dependent nuclear defects in human aging. Science 312:1059-1063. doi: 10.1126/science. 1127168

Scaffidi P, Misteli T (2008) Lamin A-dependent misregulation of adult stem cells associated with accelerated ageing. Nat Cell Biol 10:452-459. doi:10.1038/ncb1708

Shackleton S, Smallwood DT, Clayton P, Wilson LC, Agarwal AK, Garg A, Trembath RC (2005) Compound heterozygous ZMPSTE24 mutations reduce prelamin A processing and result in a severe progeroid phenotype. J Med Genet 42:e36. doi:10.1136/jmg.2004.029751

Tam A, Nouvet FJ, Fujimura-Kamada K, Slunt H, Sisodia SS, Michaelis S (1998) Dual roles for Ste24p in yeast a-factor maturation: NH2-terminal proteolysis and $\mathrm{COOH}$-terminal CAAX processing. J Cell Biol 142:635-649. doi: 10.1083/jcb.142.3.635

Toth JI, Yang SH, Qiao X, Beigneux AP, Gelb MH, Moulson CL, Miner JH, Young SG, Fong LG (2005) Blocking protein farnesyltransferase improves nuclear shape in fibroblasts from humans with progeroid syndromes. Proc Natl Acad Sci USA 102:12873-12878. doi:10.1073/pnas. 0505767102

Varela I, Cadinanos J, Pendas AM, Gutierrez-Fernandez A, Folgueras AR, Sanchez LM, Zhou Z, Rodriguez FJ, Stewart CL, Vega JA, Tryggvason K, Freije JM, LopezOtin C (2005) Accelerated ageing in mice deficient in Zmpste24 protease is linked to p53 signalling activation. Nature 437:564-568. doi:10.1038/nature04019
Varela I, Pereira S, Ugalde AP, Navarro CL, Suarez MF, Cau P, Cadinanos J, Osorio FG, Foray N, Cobo J, de Carlos F, Levy N, Freije JM, Lopez-Otin C (2008) Combined treatment with statins and aminobisphosphonates extends longevity in a mouse model of human premature aging. Nat Med 14:767-772. doi:10.1038/nm1786

Varga R, Eriksson M, Erdos MR, Olive M, Harten I, Kolodgie F, Capell BC, Cheng J, Faddah D, Perkins S, Avallone H, San H, Qu X, Ganesh S, Gordon LB, Virmani R, Wight TN, Nabel EG, Collins FS (2006) Progressive vascular smooth muscle cell defects in a mouse model of Hutchinson-Gilford progeria syndrome. Proc Natl Acad Sci USA 103:3250-3255. doi:10.1073/pnas.0600012103

Vijg J, Campisi J (2008) Puzzles, promises and a cure for ageing. Nature 454:1065-1071. doi:10.1038/nature07216

Wang Y, Panteleyev AA, Owens DM, Djabali K, Stewart CL, Worman HJ (2008) Epidermal expression of the truncated prelamin A causing Hutchinson-Gilford progeria syndrome: effects on keratinocytes, hair and skin. Hum Mol Genet 17:2357-2369. doi:10.1093/hmg/ddn136

Whyte DB, Kirschmeier P, Hockenberry TN, Nunez-Oliva I, James L, Catino JJ, Bishop WR, Pai JK (1997) K- and NRas are geranylgeranylated in cells treated with farnesyl protein transferase inhibitors. J Biol Chem 272:1445914464. doi:10.1074/jbc.272.22.14459

Willis ND, Cox TR, Rahman-Casans SF, Smits K, Przyborski SA, van den Brandt $\mathrm{P}$, van Engeland $\mathrm{M}$, Weijenberg $\mathrm{M}$, Wilson RG, de Bruine A, Hutchison CJ (2008) Lamin A/C is a risk biomarker in colorectal cancer. PLoS ONE 3:e2988. doi:10.1371/journal.pone.0002988

Yang SH, Bergo MO, Toth JI, Qiao X, Hu Y, Sandoval S, Meta M, Bendale P, Gelb MH, Young SG, Fong LG (2005) Blocking protein farnesyltransferase improves nuclear blebbing in mouse fibroblasts with a targeted HutchinsonGilford progeria syndrome mutation. Proc Natl Acad Sci USA 102:10291-10296. doi:10.1073/pnas.0504641102

Yang SH, Meta M, Qiao X, Frost D, Bauch J, Coffinier C, Majumdar S, Bergo MO, Young SG, Fong LG (2006) A farnesyltransferase inhibitor improves disease phenotypes in mice with a Hutchinson-Gilford progeria syndrome mutation. J Clin Invest 116:2115-2121. doi:10.1172/ JCI28968

Yang SH, Andres DA, Spielmann HP, Young SG, Fong LG (2008a) Progerin elicits disease phenotypes of progeria in mice whether or not it is farnesylated. J Clin Invest 118:3291-3300. doi:10.1172/JCI35876

Yang SH, Qiao X, Fong LG, Young SG (2008b) Treatment with a farnesyltransferase inhibitor improves survival in mice with a Hutchinson-Gilford progeria syndrome mutation. Biochim Biophys Acta 1781:36-39

Young SG, Meta M, Yang SH, Fong LG (2006) Prelamin A farnesylation and progeroid syndromes. J Biol Chem 281:39741-39745. doi:10.1074/jbc.R600033200

Yu CE, Oshima J, Fu YH, Wijsman EM, Hisama F, Alisch R, Matthews S, Nakura J, Miki T, Ouais S, Martin GM, Mulligan J, Schellenberg GD (1996) Positional cloning of the Werner's syndrome gene. Science 272:258-262. doi: $10.1126 /$ science. 272.5259 .258 